\title{
New Scaled Proposed formulas For Conjugate Gradient Methods in Unconstrained Optimization
}

Abbas Y. Al-Bayati

University of Telafer, Iraq

profabbasalbayati@yahoo.com

\section{ABSTRACT}

Marwan S. Jameel

University of Mosul/Iraq

Accepted on: 16/9/2013

In this paper, three efficient Scaled Nonlinear Conjugate Gradient (CG) methods for solving unconstrained optimization problems are proposed. These algorithms are implemented with inexact line searches (ILS). Powell restarting criterion is applied to all these algorithms and gives dramatic saving in the computational efficiency. The global convergence results of these algorithms are established under the Strong Wolfe line search condition. Numerical results show that our proposed CG-algorithms are efficient and stationary by comparing with standard Fletcher-Reeves (FR); PolakRibiere (PR) CG-algorithms, using 35-nonlinear test functions.

Keywords: Scaled Proposed formulas, Conjugate Gradient,Unconstrained Optimization

$$
\text { خوارزميات مقيسة جديدة للتدرج المترافق في مجال الأمثلية غير المقيدة }
$$

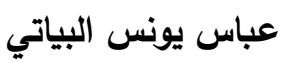

جامعة تلعفز

$$
\text { 2013/6/10 : تاريخ استلام البحث }
$$

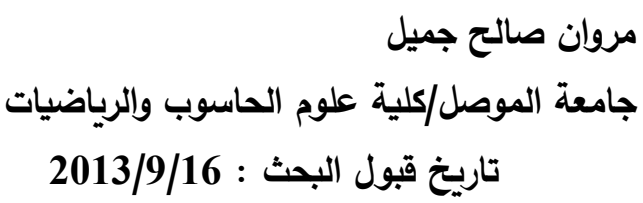

الملخص

تم في هذا البحث اقتراح ثلاث خوارزميات مقيسة جديدة في مجال الأمثلية غير المقيدة، وقد تم استخدام خط بحث غير تام ومقياس Powell للاسترجاع على جميع الصيغ المستعملة إذ يَعطي توفير جيد في الكفاءةٍ

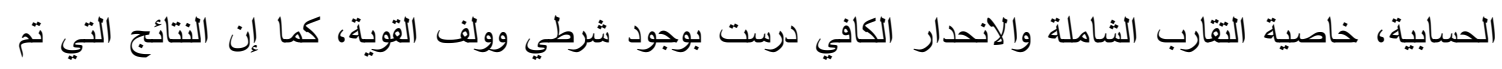
التوصل أليها عمليا أثثتت إن الخوارزميات الجديدة هي أكثر كفاءة من الخوارزميات المقارنة (FR \& PR) باستخدام 35 دالة غير خطية. الكلمات المفتاحية: خوارزميات مقيسة ، التدرج المرافق، الامثلية الغير مقيدة.

\section{Introduction}

We consider the following unconstrained optimization problem: $\min f(x), \quad x \in R^{n}$

where $f: R^{n} \rightarrow R$ is continuously differentiable and the gradient of $\mathrm{f}$ at $\mathrm{x}$ is denoted by $g(x)=\nabla f(x)$ is available. There are several kinds of numerical methods for solving equation (1), which include the Steepest Descent (SD) method; Newton method; CG and Quasi-Newton (QN) methods. Due to its simplicity and its very low memory requirement, CG-method plays a very important role, especially when the scale is large; the CG-method is very efficient. Let $x_{0} \in R^{n}$ be the initial guess of the solution of problem (1). A nonlinear CG-method is usually designed by the iterative form:[1] $x_{k+1}=x_{k}+\alpha_{k} d_{k}$

where $x_{k}$ is the current iterate point, $\alpha_{k}>0$ is a step length which is determined by some line search, and $d_{k}$ is the search direction defined by: 


$$
d_{k}= \begin{cases}-g_{k} & \text { if } \quad k=0, \\ -g_{k}+\beta_{k} d_{k-1}, & \text { if } \quad k>0,\end{cases}
$$

where $g_{k+1}$ denotes $g\left(x_{k+1}\right)$ and $\beta_{k}$ is a parameter $\left(0<\beta_{k}<1\right)$. There are some wellknown formulas for $\beta_{k}$ which are given as follows[2]:

$$
\begin{aligned}
& \beta_{k}^{F R}=\frac{g_{k+1}^{T} g_{k+1}}{g_{k}^{T} g_{k}} \\
& \beta_{k}^{H S}=\frac{g_{k+1}^{T} y_{k}}{d_{k}^{T} y_{k}} \\
& \beta_{k}^{P R}=\frac{g_{k+1}^{T} y_{k}}{g_{k}^{T} g_{k}} \\
& \beta_{k}^{C D}=\frac{g_{k+1}^{T} g_{k+1}}{-d_{k}^{T} g_{k}} \\
& \beta_{k}^{D Y}=\frac{g_{k+1}^{T} g_{k+1}}{d_{k}^{T} y_{k}} \\
& \beta_{k}^{B A 1}=\frac{y_{k}^{T} y_{k}}{-d_{k}^{T} g_{k}} \\
& \beta_{k}^{B A 2}=\frac{y_{k}^{T} y_{k}}{g_{k}^{T} g_{k}} \\
& \beta_{k}^{B A 3}=\frac{y_{k}^{T} y_{k}}{d_{k}^{T} y_{k}}
\end{aligned}
$$

(Fletcher-Reeves (FR), 1964)

(Hestenes -Stiefel (HS), 1952)

(Polak- Ribiere (PR), 1969)

(Fletcher (CD), 1987)

(Dai-Yuan (DY), 1999)

(Al-Bayati \& Al-Assady (BA1), 1986)

(Al-Bayati \& Al-Assady (BA2), 1986)

(Al-Bayati \& Al-Assady (BA3), 1986)

where $y_{k}=g_{k+1}-g_{k}$ and $\|$. $\|$ stands for the Euclidean norm of vectors. Al-Bayati and Al-Assady [3] investigated three classical CG-methods such that in numerator $y_{k}^{T} y_{k}$ and three different well-known choices for denominator as follows: $\left(-d_{k}^{T} g_{k}, g_{k}^{T} g_{k}, d_{k}^{T} y_{k}\right)$ respectively. In this paper, we have proposed three scaled CGmethods which are based on Al-Bayati and Al-Assady 's CG-methods. Generally, in the convergence analysis of CG-methods, one hopes the ILS, such as the Strong Wolfe Conditions (SWC), which is showed as follows[16]:

- The Strong Wolfe line search is to findsuch that: $\alpha_{k}$

$$
\begin{aligned}
& f\left(x_{k}+\alpha_{k} d_{k}\right) \leq f\left(x_{k}\right)+\delta \alpha_{k} g_{k}^{T} d_{k} \\
& \left|d_{k}^{T} g\left(x_{k}+\alpha_{k} d_{k}\right)\right| \leq-\sigma d_{k}^{T} g_{k} \\
& 0 \leq \delta \leq \frac{1}{2}, \text { and } \delta \leq \sigma \leq 1
\end{aligned}
$$

This paper organized as follows: In the next section, New formulas for $\beta_{k}$ with outline of our three new scaled CG-algorithms are presented. In Section 3, we have analyzed the global convergence properties for uniformly convex and general functions for the proposed new CG-methods. In Section 4, we have reported some numerical comparisons against FRCG and PRCG-methods by using 35-test problems in the CUTE [7] and general conclusions are given in Section 5. 


\section{New formulas for $\beta_{k}$}

In this section, we have constructed three New Scaled CG-Methods with the search direction $d_{k+1}$ as in (3) but $\beta_{k}$ is derived by ideology treatment of Classical AlBayati and Al-Assady (BA(1,2,3)) 's CG-methods respectively as showed in introduction and we have explained the derivation only for New1 and others are completed in same way as follows, we start from $\beta_{k}$ formula at which

$$
\beta_{k}^{B A 1}=\frac{y_{k}^{T} y_{k}}{-d_{k}^{T} g_{k}}
$$

We notice weaken this method in numerator then action some algebraic operation and positing $y_{k}=g_{k+1}-g_{k}$, we get

$$
\begin{aligned}
& \beta_{k}^{B A 1}=\frac{y_{k}^{T}\left(g_{k+1}-g_{k}\right)}{-d_{k}^{T} g_{k}} \\
& \beta_{k}^{B A 1}=\frac{y_{k}^{T} g_{k+1}-y_{k}^{T} g_{k}}{-d_{k}^{T} g_{k}}
\end{aligned}
$$

Again we set $y_{k}=g_{k+1}-g_{k}$, getting

$$
\beta_{k}^{B A 1}=\frac{g_{k+1}^{T} g_{k+1}+g_{k}^{T} g_{k}-2 g_{k+1}^{T} g_{k}}{-d_{k}^{T} g_{k}}
$$

Now, we suggest distribution parameters $(\mathrm{u}, \mathrm{v}, \mathrm{w})$ on terms existing in numerator and denominator. That is to obtaining on balance in terms after that in form $\beta_{k}$.

$$
\beta_{k}^{\text {new } 1}=\frac{u\left(\left\|g_{k+1}\right\|^{2}+\left\|g_{k}\right\|^{2}\right)-2 v g_{k+1}^{T} g_{k}}{-w d_{k}{ }^{T} g_{k}} ; 0<u, v, w \leq 1
$$

In same manner we can constricted New2 and New3 for the parameter $\beta_{k}$ thus:

$$
\begin{aligned}
& \beta_{k}^{\text {new2 }}=\frac{u\left(\left\|g_{k+1}\right\|^{2}+\left\|g_{k}\right\|^{2}\right)-2 v g_{k+1}^{T} g_{k}}{w\left\|g_{k}\right\|^{2}} ; 0<u, v, w \leq 1 \\
& \beta_{k}^{\text {new3 }}=\frac{u\left(\left\|g_{k+1}\right\|^{2}+\left\|g_{k}\right\|^{2}\right)-2 v g_{k+1}^{T} g_{k}}{w d_{k}^{T} y_{k}} ; 0<u, v, w \leq 1
\end{aligned}
$$

\subsection{Outline of the Three New Scaled CG-Algorithms}

Step1: (Initializing). Given an initial point $x_{0} \in R^{n}$ and positive parameters, $0<u, v, w \leq 1, \psi=0.2,0 \leq \delta \leq 0.5$ and $\delta \leq \sigma \leq 1$. Set the initial search direction $d_{0}=-g_{0}$ and Let $\mathrm{k}=0$.

Step2: (Termination Criterion). If $\left\|g_{k}\right\| \leq \varepsilon$, then stop.

Step3: (Line search). Determine step length $\alpha_{k}>0$ satisfying the Strong Wolfe

Condition (4) with Acceleration scheme[5]: compute

$z=x_{k}+\alpha_{k} d_{k}, y_{k}=g_{k}-g_{z}, . g_{z}=\nabla f(z)$

And Compute $a_{k}=\alpha_{k} g_{k}^{T} d_{k}, b_{k}=-\alpha_{k} y_{k}^{T} d_{k}$, if $b_{k} \neq 0$, then compute: 
$\varphi_{k}=-\frac{a_{k}}{b_{k}}$ and update the variables as $x_{k+1}=x_{k}+\varphi_{k} \alpha_{k} d_{k}$; otherwise update the variables as $x_{k+1}=x_{k}+\alpha_{k} d_{k}$.

Step4: (Finding the direction). Compute the new search direction $d_{k+1}=-g_{k+1}+\beta_{k}^{\text {new }} d_{k}$, where the scalar parameters $\beta_{k}^{\text {new }}$ are known in (5), (6) and (7).

Step5: (Restart procedure ). If $\left|g_{k+1}^{T} g_{k}\right| \geq \psi\left\|g_{k+1}\right\|^{2}$, then go to Step (1) else continue.(this is Powell restart).[14]

Step6: (Loop). Let $\mathrm{k}=\mathrm{k}+1$ and go to Step (2).

\section{Convergence Analysis.}

Now, we have to prove the global convergence property of these three new CGalgorithms under the condition that the following assumption is hold.

\section{Assumption (H)}

(i) The level set $S=\left\{x: x \in R^{n}, f(x) \leq f\left(x_{0}\right)\right\}$ is bounded, where $x_{0}$ is the starting point.

(ii) In a neighborhood $\Omega$ of $\mathrm{S}, f$ is continuously differentiable and its gradient $g$ is Lipschitz continuously, namely, there exists a constant $L \geq 0$ such that $\left\|\mathrm{g}(\mathrm{x})-\mathrm{g}\left(\mathrm{x}_{\mathrm{k}}\right)\right\| \leq \mathrm{L}\left\|\mathrm{x}-\mathrm{x}_{\mathrm{k}}\right\|, \forall \mathrm{x}, \mathrm{x}_{\mathrm{k}} \in \Omega$

Obviously, from the Assumption $(\mathrm{H}, \mathrm{i})$ there exists a positive constant $\mathrm{D}$ such that:

$D=\max \left\{\left\|x-x_{k}\right\|, \forall x, x_{k} \in S\right\}$

where $\mathrm{D}$ is the diameter of $\Omega$. From Assumption (H, ii), we also know that there exists a constant $\Gamma \geq 0$, such that:

$\|g(x)\| \leq \gamma, \forall x \in S$

On some studies of the CG-methods, the sufficient descent or descent condition plays an important role, but unfortunately some times, this condition is hard to hold.[16]

3.1. Theorem Suppose that Assumption (H) holds and satisfies the SWC (4). consider any CG-method (2)-(3) with scalar parameter $\beta_{k}$ is defined in (5)-(7) respectively are satisfies the Sufficient Descent condition with:

$$
\begin{aligned}
& c_{1}=\left\{\left(1-\frac{2 v \sigma \psi+u \sigma+u \psi}{w}\right)\right\} \\
& c_{2}=\left\{\left(1-\frac{c(u \sigma+u \psi+2 v \sigma \psi)}{w}\right)\right\} \\
& c_{3}=\left\{\left(1-\frac{u \sigma-u \psi+2 v \psi \sigma}{w \sigma}\right)\right\} \\
& 0<u, v, \sigma, \psi<w \leq 1
\end{aligned}
$$

\section{Proof}

Case (1) we have to prove that the CG-method $d_{k+1}$ from (3) with (5) and multiplying by $g_{k+1}$, also put value of $\beta_{k}^{\text {newl }}$, we get: 
$d_{k+1}{ }^{T} g_{k+1}=-\left\|g_{k+1}\right\|^{2}+\left[\frac{u\left(\left\|g_{k+1}\right\|^{2}+\left\|g_{k}\right\|^{2}\right)-2 v g_{k+1}^{T} g_{k}}{-w d_{k}^{T} g_{k}}\right] d_{k}^{T} g_{k+1}$

We obtain from (4)

$\sigma d_{k}^{T} g_{k} \leq d_{k}^{T} g_{k+1} \leq-\sigma d_{k}^{T} g_{k}$

Since the Powell restarting criterion is defined as follows:

$\left|g_{k+1}^{T} g_{k}\right| \geq \psi\left\|g_{k+1}\right\|^{2}$

Then we get:

$g_{k+1}^{T} g_{k} \leq-\psi\left\|g_{k+1}\right\|^{2}$

Using (12) and (14) in the inequality (11) become:

$$
\begin{aligned}
d_{k+1}^{T} g_{k+1} & =-\left\|g_{k+1}\right\|^{2}-\frac{u\left\|g_{k+1}\right\|^{2}}{w d_{k}{ }^{T} g_{k}} d_{k}{ }^{T} g_{k+1}-\frac{u\left\|g_{k}\right\|^{2}}{w d_{k}{ }^{T} g_{k}} d_{k}{ }^{T} g_{k+1}+\frac{2 v g_{k+1}{ }^{T} g_{k}}{w d_{k}{ }^{T} g_{k}} d_{k}{ }^{T} g_{k+1} \\
& =-\left\|g_{k+1}\right\|^{2}-\frac{u d_{k}{ }^{T} g_{k+1}}{w d_{k}{ }^{T} g_{k}}\left\|g_{k+1}\right\|^{2}-\frac{u d_{k}{ }^{T} g_{k}}{w d_{k}{ }^{T} g_{k}} g_{k+1}{ }^{T} g_{k}+\frac{2 v d_{k}{ }^{T} g_{k+1}}{w d_{k}{ }^{T} g_{k}} g_{k+1}{ }^{T} g_{k} \\
& \leq-\left\|g_{k+1}\right\|^{2}+\frac{u \sigma d_{k}{ }^{T} g_{k}}{w d_{k}{ }^{T} g_{k}}\left\|g_{k+1}\right\|^{2}+\frac{u \psi}{w}\left\|g_{k+1}\right\|^{2}+\frac{2 v \sigma \psi d_{k}{ }^{T} g_{k}}{w d_{k}{ }^{T} g_{k}}\left\|g_{k+1}\right\|^{2} \\
& \leq\left(-1+\frac{2 v \sigma \psi}{w}+\frac{u \sigma}{w}+\frac{u \psi}{w}\right)\left\|g_{k+1}\right\|^{2}
\end{aligned}
$$

Dividing by $\left\|g_{k+1}\right\|^{2}$, we get:

$\frac{d_{k+1}{ }^{T} g_{k+1}}{\left\|g_{k+1}\right\|^{2}} \leq-\left\{\left(1-\frac{2 v \sigma \psi+u \sigma+u \psi}{w}\right)\right\}=-c_{1}$

Hence the sufficient descent condition hold, i.e.

$d_{k+1}{ }^{T} g_{k+1} \leq-c_{1}\left\|g_{k+1}\right\|^{2}, c_{1} \geq 0$

$0<u, v, \sigma, \psi<w \leq 1$.

Case (2) take $d_{k+1}$ from (3) with (6) proceed by induction. For $k=1$ we have:

$$
d_{1}=-g_{1}
$$

and

$$
d_{1}^{T} g_{1}=-g_{1}^{T} g_{1}=-\left\|g_{1}\right\|^{2} \leq 0
$$

suppose that:

$d_{k}^{T} g_{k} \leq-c\left\|g_{k}\right\|^{2}$

Multiplying the new search direction by $g_{k+1}$ and put value of $\beta_{k}^{\text {new2 }}$, we get:

$$
\begin{aligned}
d_{k+1}{ }^{T} g_{k+1} & =-\left\|g_{k+1}\right\|^{2}+\left[\frac{u\left(\left\|g_{k+1}\right\|^{2}+\left\|g_{k}\right\|^{2}\right)-2 v g_{k+1}^{T} g_{k}}{w\left\|g_{k}\right\|^{2}}\right] d_{k}^{T} g_{k+1} \\
& =-\left\|g_{k+1}\right\|^{2}+\frac{u\left\|g_{k+1}\right\|^{2}}{w\left\|g_{k}\right\|^{2}} d_{k}^{T} g_{k+1}+\frac{u\left\|g_{k}\right\|^{2}}{w\left\|g_{k}\right\|^{2}} d_{k}^{T} g_{k+1}-\frac{2 v g_{k+1}{ }^{T} g_{k}}{w\left\|g_{k}\right\|^{2}} d_{k}{ }^{T} g_{k+1} \\
& =-\left\|g_{k+1}\right\|^{2}+\frac{u d_{k}{ }^{T} g_{k+1}}{w\left\|g_{k}\right\|^{2}}\left\|g_{k+1}\right\|^{2}+\frac{u d_{k}{ }^{T} g_{k}}{w\left\|g_{k}\right\|^{2}} g_{k+1}{ }^{T} g_{k}-\frac{2 v d_{k}{ }^{T} g_{k+1}}{w\left\|g_{k}\right\|^{2}} g_{k+1}{ }^{T} g_{k}
\end{aligned}
$$


Using strong Wolfe condition (4) and the Powell restarting condition (14) in the above inequality we obtain:

$$
\begin{aligned}
d_{k+1}{ }^{T} g_{k+1} & \leq-\left\|g_{k+1}\right\|^{2}-\frac{u \sigma d_{k}{ }^{T} g_{k}}{w\left\|g_{k}\right\|^{2}}\left\|g_{k+1}\right\|^{2}-\frac{u \psi d_{k}{ }^{T} g_{k}}{w\left\|g_{k}\right\|^{2}}\left\|g_{k+1}\right\|^{2}-\frac{2 v \sigma \psi d_{k}{ }^{T} g_{k}}{w\left\|g_{k}\right\|^{2}}\left\|g_{k+1}\right\|^{2} \\
& \leq-\left\|g_{k+1}\right\|^{2}-\left(\frac{u \sigma+u \psi+2 v \sigma \psi}{w}\right) \frac{\left\|g_{k+1}\right\|^{2}}{\left\|g_{k}\right\|^{2}} d_{k}{ }^{T} g_{k} \\
\frac{d_{k+1}{ }^{T} g_{k+1}}{\left\|g_{k+1}\right\|^{2}} & \leq-\left(1-\frac{c(u \sigma+u \psi+2 v \sigma \psi)}{w}\right)=-c_{2}
\end{aligned}
$$

Hence the sufficient descent condition hold, i.e.

$$
\begin{aligned}
& d_{k+1}{ }^{T} g_{k+1} \leq-c_{2}\left\|g_{k+1}\right\|^{2}, c_{2} \geq 0 \\
& 0<u, v, \sigma, \psi<w \leq 1 .
\end{aligned}
$$

Case (3) Also, take $d_{k+1}$ from (3) with (7) and multiplying by $g_{k+1}$ with the value of $\beta_{k}^{\text {new3 }}$ to get:

$$
d_{k+1}{ }^{T} g_{k+1}=-\left\|g_{k+1}\right\|^{2}+\left[\frac{u\left(\left\|g_{k+1}\right\|^{2}+\left\|g_{k}\right\|^{2}\right)-2 v g_{k}^{T} g_{k}}{w d_{k}{ }^{T} y_{k}}\right] d_{k}{ }^{T} g_{k+1}
$$

But:

$$
\begin{aligned}
d_{k}{ }^{T} y_{k}=d_{k}{ }^{T} & g_{k+1}-d_{k}{ }^{T} g_{k} \geq d_{k}{ }^{T} g_{k+1} \\
& \Rightarrow d_{k}{ }^{T} y_{k} \geq d_{k}{ }^{T} g_{k+1} \\
& \Rightarrow \frac{1}{w d_{k}{ }^{T} y_{k}} \leq \frac{1}{w d_{k}{ }^{T} g_{k+1}}
\end{aligned}
$$

Putting (19) in (18) yields:

$$
\begin{aligned}
& d_{k+1}^{T} g_{k+1} \leq-\left\|g_{k+1}\right\|^{2}+\left[\frac{u\left(\left\|g_{k+1}\right\|^{2}+\left\|g_{k}\right\|^{2}\right)-2 v g_{k+1}^{T} g_{k}}{w d_{k}^{T} g_{k+1}}\right] d_{k}^{T} g_{k+1} \\
& \leq-\left\|g_{k+1}\right\|^{2}+\frac{u\left\|g_{k+1}\right\|^{2}}{w d_{k}{ }^{T} g_{k+1}} d_{k}^{T} g_{k+1}+\frac{u\left\|g_{k}\right\|^{2}}{w d_{k}{ }^{T} g_{k+1}} d_{k}{ }^{T} g_{k+1}-\frac{2 v g_{k+1}{ }^{T} g_{k}}{w d_{k}{ }^{T} g_{k+1}} d_{k}{ }^{T} g_{k+1} \\
& \leq-\left\|g_{k+1}\right\|^{2}+\frac{u d_{k}{ }^{T} g_{k+1}}{w d_{k}{ }^{T} g_{k+1}}\left\|g_{k+1}\right\|^{2}+\frac{u d_{k}{ }^{T} g_{k}}{w d_{k}{ }^{T} g_{k+1}} g_{k+1}{ }^{T} g_{k}-\frac{2 v d_{k}{ }^{T} g_{k+1}}{w d_{k}{ }^{T} g_{k+1}} g_{k+1}^{T} g_{k}
\end{aligned}
$$

Using (4) and (14) :

$$
\begin{aligned}
d_{k+1}^{T} g_{k+1} & \leq-\left\|g_{k+1}\right\|^{2}+\frac{u}{w}\left\|g_{k+1}\right\|^{2}+\frac{u d_{k}^{T} g_{k+1}}{w \sigma d_{k}^{T} g_{k+1}}\left(-\psi\left\|g_{k+1}\right\|^{2}\right)-\frac{2 v d_{k}^{T} g_{k+1}}{w d_{k}{ }^{T} g_{k+1}}\left(-\psi\left\|g_{k+1}\right\|^{2}\right) \\
& \leq-\left\|g_{k+1}\right\|^{2}+\frac{u}{w}\left\|g_{k+1}\right\|^{2}-\frac{u \psi}{w \sigma}\left\|g_{k+1}\right\|^{2}+\frac{2 v \psi}{w}\left\|g_{k+1}\right\|^{2}
\end{aligned}
$$

Dividing this inequality by $\left\|g_{k+1}\right\|^{2}$ yields:

$$
\frac{d_{k+1}{ }^{T} g_{k+1}}{\left\|g_{k+1}\right\|^{2}} \leq-\left(1-\frac{u \sigma-u \psi+2 v \psi \sigma}{w \sigma}\right)=-c_{3}
$$


Hence the sufficient descent hold i.e.

$d_{k+1}{ }^{T} g_{k+1} \leq-c_{3}\left\|g_{k+1}\right\|^{2}, \quad c_{3} \geq 0$

$0<\{u, v, w, \sigma, \psi\} \leq 1$

3.2. Property Consider a general CG-method and suppose that[12]:

$0<\varsigma \leq\left\|g_{k}\right\| \leq \gamma, \quad \forall k \geq 0$

we say that a CG-method has the Property (3.2), if there exists two constants $b>1$ and $\lambda>0$ such that for all $\mathrm{k}$,

$\left|\beta_{k}^{\text {new }}\right| \leq b$

If $\left\|s_{k}\right\| \leq \lambda$ then $\left|\beta_{k}^{\text {new }}\right| \leq \frac{1}{2 b}$ for all $\lambda>0$

3.3. Lemma Suppose that Assumption $(\mathrm{H})$ hold. If there exists a constant $\varsigma>0$ such that $\left\|g_{k}\right\| \geq \varsigma$, for all positive $\mathrm{k}$, then the following holds. If $d_{k}$ is satisfies the sufficient descent condition $\left(g_{k}^{T} d_{k} \leq-c\left\|g_{k}\right\|^{2}, \forall k \geq 0\right)$ and $\alpha_{k}$ is obtained by (4). The parameters $\left(\beta_{k}^{\text {new } 1}, \beta_{k}^{\text {new } 2}, \beta_{k}^{\text {new3 }}\right.$ ) in our CG-methods satisfy Property (3.2).

\section{Proof}

First we can prove this property for the first algorithm with the parameter(5):

$$
\begin{aligned}
\beta_{k}^{\text {new } 1} & =\frac{u\left(\left\|g_{k+1}\right\|^{2}+\left\|g_{k}\right\|^{2}\right)-2 v g_{k+1}^{T} g_{k}}{-w d_{k}^{T} g_{k}} \quad 0<u, v, w \leq 1 \\
\left|\beta_{k}^{\text {new } 1}\right| \leq \frac{u\left(\left\|g_{k+1}\right\|^{2}+\left\|g_{k}\right\|^{2}\right)+2 v\left\|g_{k+1} \mid\right\| g_{k} \|}{w\left|-d_{k}{ }^{T} g_{k}\right|} &
\end{aligned}
$$

From (15) we have:

$$
\begin{aligned}
& \frac{1}{d_{k}^{T} g_{k}} \geq \frac{1}{-c\left\|g_{k}\right\|^{2}} \\
& \Rightarrow \frac{1}{-d_{k}^{T} g_{k}} \leq \frac{1}{c\left\|g_{k}\right\|^{2}} \\
& \Rightarrow \frac{1}{\left|-d_{k}^{T} g_{k}\right|} \leq \frac{1}{c\left\|g_{k}\right\|^{2}}
\end{aligned}
$$

After putting (26) in (25) we get:

$$
\begin{gathered}
\left|\beta_{k}^{\text {new1 }}\right| \leq \frac{u\left(\left\|g_{k+1}\right\|^{2}+\left\|g_{k}\right\|^{2}\right)+2 v\left\|g_{k+1} \mid\right\| g_{k} \|}{w c\left\|g_{k}\right\|^{2}} \\
\leq \frac{u\left(\gamma^{2}+\gamma^{2}\right)+2 v \gamma^{2}}{w c \gamma^{2}} \\
=\frac{2(u+v)}{w c}=b_{1}=\frac{2 u \gamma^{2}+2 v \gamma^{2}}{w c \gamma^{2}}
\end{gathered}
$$

Now, let us define: 
$\lambda_{1}=\frac{8(u+v)^{2} \alpha \gamma}{w^{2} c}$ and $\left\|s_{k}\right\| \leq \lambda_{1}, \lambda_{1} \geq 0$

And from this relation, we have:

$d_{k}^{T} g_{k}=\frac{1}{\alpha} s_{k}^{T} g_{k}$

By using (25) and (28) with the value of $\lambda$, we get:

$$
\begin{aligned}
\left|\beta_{k}^{\text {new } 1}\right| & \leq \frac{u\left(\left\|g_{k+1}\right\|^{2}+\left\|g_{k}\right\|^{2}\right)+2 v\left\|g_{k+1}\right\|\left\|g_{k}\right\|}{\frac{w}{\alpha}\left\|s_{k}\right\|\left\|g_{k}\right\|} \\
& \leq \frac{2(u+v) \alpha \gamma^{2}}{w \gamma \lambda_{1}}=\frac{2(u+v) \alpha \gamma}{w \lambda_{1}}=\frac{1}{2 b_{1}}
\end{aligned}
$$

Hence

$\left|\beta_{k}^{\text {new } 1}\right| \leq \frac{1}{2 b_{1}} \quad$ when $\quad\left\|s_{k}\right\| \leq \lambda_{1}$.

Second similarity, as in the first proof, we will deal with the new second algorithm as defined in (6):

$$
\begin{aligned}
\beta_{k}^{\text {new } 2} & =\frac{u\left(\left\|g_{k+1}\right\|^{2}+\left\|g_{k}\right\|^{2}\right)-2 v g_{k+1}{ }^{T} g_{k}}{w\left\|g_{k}\right\|^{2}} \quad 0<u, v, w \leq 1 \\
\left|\beta_{k}^{\text {new } 2}\right| & \leq \frac{u\left(\left\|g_{k+1}\right\|^{2}+\left\|g_{k}\right\|^{2}\right)+2 v\left\|g_{k+1}\right\|\left\|g_{k}\right\|}{w\left\|g_{k}\right\|^{2}} \\
& \leq \frac{u \gamma^{2}+u \gamma^{2}+2 v \gamma^{2}}{w \gamma^{2}}=\frac{2(u+v)}{w}=b_{2}
\end{aligned}
$$

Now, again let us define:

$\lambda_{2}=\frac{8(u+v)^{2} \alpha c \gamma^{2}}{w^{2}} \quad$ and $\quad\left\|s_{k}\right\| \leq \lambda_{2},\left(\lambda_{2}>0\right)$

Now from the descent property (20) we have:

$$
\begin{aligned}
& d_{k}^{T} g_{k} \leq-c\left\|g_{k}\right\|^{2} \\
& \Rightarrow\left|d_{k}^{T} g_{k}\right| \leq c\left\|g_{k}\right\|^{2} \\
& \Rightarrow \frac{1}{c\left\|g_{k}\right\|^{2}} \leq \frac{1}{\left|d_{k}^{T} g_{k}\right|} \leq \frac{1}{\left\|d_{k}\right\|\left\|g_{k}\right\|} \\
& \left|\beta_{k}^{\text {new } 2}\right| \leq \frac{u\left(\left\|g_{k+1}\right\|^{2}+\left\|g_{k}\right\|^{2}\right)+2 v\left\|g_{k+1} \mid\right\| g_{k} \|}{\frac{w}{\alpha c}\left\|s_{k} \mid\right\| g_{k} \|} \\
& \quad \leq \frac{2 \alpha c(u+v) \gamma^{2}}{w \lambda_{2} \gamma}=\frac{2 \alpha c(u+v) \gamma}{w \lambda_{2}}=\frac{1}{2 b_{2}}
\end{aligned}
$$

Hence:

$\left|\beta_{k}^{\text {new } 1}\right| \leq \frac{1}{2 b_{2}} ;$ when $\left\|s_{k}\right\| \leq \lambda_{2}$. 
Third let us again try as in the last proof with the third algorithm (7) as:

$\beta_{k}^{\text {new } 3}=\frac{u\left(\left\|g_{k+1}\right\|^{2}+\left\|g_{k}\right\|^{2}\right)-2 v g_{k}^{T} g_{k}}{w d_{k}^{T} y_{k}}$

by utilize from (4) to get:

$$
\begin{aligned}
& \sigma d_{k}{ }^{T} g_{k} \leq d_{k}{ }^{T} g_{k+1} \leq-\sigma d_{k}^{T} g \\
& \Rightarrow d_{k}^{T} g_{k+1}-d_{k}^{T} g_{k} \geq \sigma d_{k}^{T} g_{k}-d_{k}^{T} g_{k} \\
& \Rightarrow d_{k}^{T} y_{k} \geq-(1-\sigma) d_{k}{ }^{T} g_{k}
\end{aligned}
$$

By adding to $-d_{k}^{T} g_{k} \geq c\left\|g_{k}\right\|^{2}$ then (30) becomes:

$\Rightarrow d_{k}{ }^{T} y_{k} \geq c(1-\sigma)\left\|g_{k}\right\|^{2}$

Taking the absolute values of (30) and since $d_{k}^{T} y_{k}=\frac{1}{\alpha} s_{k}^{T} y_{k}$ then:

$$
\left|\beta_{k}^{\text {new3 }}\right| \leq \frac{u\left(\left\|g_{k+1}\right\|^{2}+\left\|g_{k}\right\|^{2}\right)+2 v\left\|g _ { k + 1 } \left|\left\|\mid g_{k}\right\|\right.\right.}{\frac{w}{\alpha}\left|s_{k}{ }^{T} y_{k}\right|}
$$

Using inequality (32) the above inequality yields:

$$
\begin{aligned}
\left|\beta_{k}^{\text {new } 3}\right| & \leq \frac{u\left(\left\|g_{k+1}^{T}\right\|^{2}+\left\|g_{k}\right\|^{2}\right)+2 v\left\|g_{k+1} \mid\right\| g_{k} \|}{w c(1-\sigma)\left\|g_{k}\right\|^{2}} \\
& \leq \frac{2(u+v) \gamma^{2}}{w(1-\sigma) \gamma^{2}}=\frac{2(u+v)}{w(1-\sigma)}=b_{3}
\end{aligned}
$$

Now, also let us define:

$$
\begin{gathered}
\lambda_{3}=\frac{8 \alpha(u+v)^{2} \gamma}{w^{2}(1-\sigma)^{2}} \text { and }\left\|s_{k}\right\| \leq \lambda_{3},\left(\lambda_{3}>0\right) \\
\left|\beta_{k}^{\text {new } 3}\right| \leq \frac{u\left(\left\|g_{k+1}\right\|^{2}+\left\|g_{k}\right\|^{2}\right)+2 v\left\|g_{k+1} \mid\right\| g_{k} \|}{\mid \frac{w}{\alpha}\left(-(1-\sigma)\left|\left\|s_{k} \mid\right\| g_{k} \|\right)\right.} \\
\leq \frac{2 \alpha(u+v) \gamma^{2}}{w(1-\sigma) \lambda_{3} \gamma}=\frac{2 \alpha(u+v) \gamma}{w(1-\sigma) \lambda_{3}}=\frac{1}{2 b_{3}}
\end{gathered}
$$

Hence

$\left|\beta_{k}^{\text {new } 3}\right| \leq \frac{1}{2 b_{3}} \quad ;$ when $\left\|s_{k}\right\| \leq \lambda_{3}$

3.4. Lemma Assume that $d_{k+1}$ is a descent direction and $g_{k}$ satisfies the Lipschitz condition $\left\|g(x)-g\left(x_{k}\right)\right\| \leq L\left\|x-x_{k}\right\|$ for all $x$ on the line segment connecting $x$ and $x_{k}$, where $\mathrm{L}$ is constant If the line search direction satisfy (4), then[6]:

$$
\alpha_{k} \geq \frac{(1-\sigma)\left|d_{k}{ }^{T} g_{k}\right|}{L\left\|d_{k}\right\|^{2}}
$$

Proof Using curvature inequality in (4)

$$
\sigma d_{k}^{T} g_{k} \leq d_{k}^{T} g_{k+1} \leq-\sigma d_{k}^{T} g_{k}
$$


$\Rightarrow \sigma d_{k}^{T} g_{k} \leq d_{k}^{T} g_{k+1}$

Subtracting $d_{k}{ }^{T} g_{k}$ from both sides of (35) and using Lipschitz condition yields: $(1-\sigma) d_{k}^{T} g_{k} \leq d_{k}^{T}\left(g_{k+1}-g_{k}\right) \leq L \alpha_{k}\left\|d_{k}\right\|^{2}$

Since $d_{k}$ is descent direction and $\sigma \leq 1$, then (34) holds:

$$
\alpha_{k} \geq \frac{(1-\sigma)\left|d_{k}{ }^{T} g_{k}\right|}{L\left\|d_{k}\right\|^{2}}
$$

The conclusion of the following Lemma, often called the Zoutendijk condition is used to prove the global convergence of any nonlinear CG-method. It was originally given by Zoutendijk [18] under the Strong Wolfe line search (4). In following Lemma, we will prove this condition.

3.5. Lemma Suppose Assumption $(\mathrm{H})$ holds. Consider the iteration process of the form (2)-(3), where $d_{k+1}$ satisfies the descent condition ( $d_{k}{ }^{T} g_{k} \leq \mathrm{O}$ ) for all $k \geq 1$ and $\alpha_{k}$ satisfies (4). Then

$\sum_{k \geq 1} \frac{\left(g_{k}{ }^{T} d_{k}\right)^{2}}{\left\|d_{k}\right\|^{2}}<+\infty$

Proof From the first inequality in (4) we can get:

$$
f_{k+1}-f_{k} \leq \delta \alpha_{k} g_{k}^{T} d_{k}
$$

Combining this with the results in Lemma (3.4), yields

$f_{k+1}-f_{k} \leq \frac{\delta(1-\sigma)}{L} \frac{\left(g_{k}{ }^{T} d_{k}\right)^{2}}{\left\|d_{k}\right\|^{2}}$

Using the bound-ness of function $\mathrm{f}$ in Assumption $(\mathrm{H})$, hence

$\sum_{k \geq 1} \frac{\left(g_{k}{ }^{T} d_{k}\right)^{2}}{\left\|d_{k}\right\|^{2}}<+\infty$

\subsection{Global Convergence Property For Uniformly Convex Functions}

Under Assumption $(\mathrm{H})$ on $f$, there exists a constant $\gamma \geq 0$, such that $\|\nabla f(x)\| \leq \gamma$, for all $x \in S$, then for any CG-method with Strong Wolfe line search, the following general result holds.[8]

3.6.1. Theorem Let Assumption (H) holds and consider any CG-method (2)-(3), where $d_{k+1}$ is a descent direction and $\alpha_{k}$ is obtained by (4) line search, if

$\sum_{k \geq 1} \frac{1}{\left\|d_{k}\right\|^{2}}=\infty$

Then

$\liminf _{k \rightarrow \infty}\left\|g_{k}\right\|=0$

For uniformly convex function which satisfied the above assumptions we can prove that the norm of $d_{k+1}$ given by (3) with (5), (6) and (7) is bounded above. Assume 
that the function $f$ is uniformly convex function, i.e. there exists a constant $\mu \geq 0$ such that for all $x, x_{k} \in S$

$$
\left(g(x)-g\left(x_{k}\right)\right)^{T}\left(x-x_{k}\right) \geq \mu\left\|x-x_{k}\right\|^{2}
$$

and the step-length $\alpha_{k}$ is obtained by the Strong Wolfe line search (4), Now try to prove the following result:

3.6.2. Theorem Suppose that Assumption $(H)$ hold. Consider the algorithm (2.1), where $0<u, v, w \leq 1$, for $\gamma>0$, let $\left\|g_{k}\right\| \leq \gamma,\left\|g_{k+1}\right\| \leq \gamma$ and $\alpha_{k}$ is obtained by (SWC) line search. If $\left\|s_{k}\right\|$ tends to zero and there exists non-negative constants $\eta_{1}$ and $\eta_{2}$ such that[6]:

$$
\left\|g_{k}\right\|^{2} \geq \eta_{1}\left\|s_{k}\right\|^{2} ;\left\|g_{k+1}\right\|^{2} \leq \eta_{2}\left\|s_{k}\right\|
$$

and if $f$ is a uniformly convex function, then:

$\operatorname{Lim}_{k \rightarrow \infty} g_{k}=0$

\section{Proof}

Case(1) we have from (5) and if we taking the absolute value:

$$
\begin{aligned}
\left|\beta_{k+1}^{\text {new } 1}\right| & \leq \frac{u\left(\left\|g_{k+1}\right\|^{2}+\left\|g_{k}\right\|^{2}\right)+2 v\left\|g_{k+1}\right\|\left\|g_{k}\right\|}{\frac{w}{\alpha_{k}}\left\|s_{k}\right\|\left\|g_{k}\right\|} \\
& \leq \frac{u \eta_{2}\left\|s_{k}\right\|+u \eta_{1}\left\|s_{k}\right\|^{2}+2 v \gamma}{\frac{w}{\alpha_{k}} \xi\left\|s_{k}\right\|}
\end{aligned}
$$

But $\left\|S_{k}\right\|=\left\|x-x_{k}\right\|$ and since $D=\max \left\{\left\|x-x_{k}\right\|, \forall x, x_{k} \in S\right\}$ is diameter of the level set $\mathrm{S}$ (9), then

$$
\left|\beta_{k}^{\text {new } 1}\right| \leq \frac{u\left(\eta_{2}+\eta_{1} D\right) D+2 v \gamma^{2}}{\frac{w}{\alpha_{k}} \xi D}
$$

Taking the norm and square both sides of the new search direction, we get:

$$
\begin{aligned}
& \left\|d_{k+1}\right\|^{2}=\left\|-g_{k+1}+\beta_{k}^{\text {new } 1} d_{k}\right\|^{2} \\
& \leq\left\|g_{k+1}\right\|^{2}+2 \mid \beta_{k}^{\text {new1 } 1}\left\|g_{k+1}\right\| d_{k}\left\|+\left(\beta_{k}^{\text {new } 1}\right)^{2}\right\| d_{k} \|^{2} \\
& \leq \eta_{2}\left\|s_{k}\right\|+2\left(\frac{u\left(\eta_{2}+\eta_{1} D\right) D+2 v \gamma^{2}}{\frac{w}{\alpha_{k}} \xi D}\right) \frac{1}{\alpha_{k}} \gamma\left\|s_{k}\right\|+\left(\frac{u\left(\eta_{2}+\eta_{1} D\right) D+2 v \gamma^{2}}{\frac{w}{\alpha_{k}} \xi D}\right)^{2} \frac{1}{\alpha_{k}^{2}}\left\|s_{k}\right\|^{2} \\
& \leq \eta_{2}\left\|s_{k}\right\|+2\left(\frac{u\left(\eta_{2}+\eta_{1} D\right) D+2 v \gamma^{2}}{\frac{w}{\alpha_{k}} \xi D}\right) \frac{1}{\alpha_{k}} \gamma\left\|s_{k}\right\|+\left(\frac{u\left(\eta_{2}+\eta_{1} D\right) D+2 v \gamma^{2}}{\frac{w}{\alpha_{k}} \xi D}\right)^{2} \frac{1}{\alpha_{k}^{2}}\left\|s_{k}\right\|^{2} \\
& \leq \eta_{2}\left\|s_{k}\right\|+2\left(\frac{u\left(\eta_{2}+\eta_{1} D\right) D+2 v \gamma^{2}}{w \xi D}\right) \gamma\left\|s_{k}\right\|+\left(\frac{u\left(\eta_{2}+\eta_{1} D\right) D+2 v \gamma^{2}}{w \xi D}\right)^{2}\left\|s_{k}\right\|^{2}
\end{aligned}
$$




$$
\leq \eta_{2} D+2\left(\frac{u\left(\eta_{2}+\eta_{1} D\right) D+2 v \gamma^{2}}{w \xi}\right) \gamma+\left(\frac{u\left(\eta_{2}+\eta_{1} D\right) D+2 v \gamma^{2}}{w \xi}\right)^{2}=\psi_{1}^{2}
$$

Thus $\left\|d_{k+1}\right\|^{2} \leq \psi_{1}^{2}$

$$
\Rightarrow \frac{1}{\left\|d_{k+1}\right\|^{2}} \geq \frac{1}{\psi_{1}^{2}}
$$

Summation this inequality for all $k \geq 1$

$$
\sum_{k \geq 1} \frac{1}{\left\|d_{k+1}\right\|^{2}} \geq \sum_{k \geq 1} \frac{1}{\psi_{1}^{2}}=\infty
$$

Using Theorem (3.2.1), hence $\liminf _{k \rightarrow \infty}\left\|g_{k}\right\|=0$

But $f$ is uniformly convex (3.37) therefore satisfies $\lim _{k \rightarrow \infty} g_{k}=0$.

Case (2) similarly we begin taking the absolute to the second scalar parameter $\beta_{k}^{\text {new } 2}$

$$
\begin{aligned}
\left|\beta_{k}^{\text {new } 2}\right| & \leq \frac{u\left\|g_{k+1}\right\|^{2}+u\left\|g_{k}\right\|^{2}+2 v\left\|g_{k+1} \mid\right\| g_{k} \|}{w\left\|g_{k}\right\|^{2}} \\
\leq & \frac{u \eta_{2}\left\|s_{k}\right\|+u \eta_{1}\left\|s_{k}\right\|^{2}+2 v \gamma \gamma}{w \eta_{1}\left\|s_{k}\right\|^{2}} \\
& \leq \frac{u\left(\eta_{2}+\eta_{1} D\right) D+2 v \gamma^{2}}{w \eta_{1} D^{2}}
\end{aligned}
$$

where $\mathrm{D}$ is a diameter of the level set $\mathrm{S}$ and the new direction can be evaluated from (3) with (6). Now since:

$$
\begin{aligned}
& d_{k+1}=-g_{k+1}+\beta_{k}^{\text {new } 2} d_{k} \\
\left\|d_{k+1}\right\|^{2} & =\left\|-g_{k+1}+\beta_{k}^{\text {new } 2} d_{k}\right\|^{2} \\
\leq & \left\|g_{k+1}\right\|^{2}+2 \mid \beta_{k}^{\text {new } 2}\left\|g_{k+1}\right\| d_{k}\left\|+\left(\beta_{k}^{\text {new } 2}\right)^{2}\right\| d_{k} \|^{2} \\
\leq & \eta_{2}\left\|s_{k}\right\|+2\left(\frac{u\left(\eta_{2}+\eta_{1}\left\|s_{k}\right\|\right)\left\|s_{k}\right\|+2 v \gamma^{2}}{w \eta_{1}\left\|s_{k}\right\|^{2}}\right) \frac{\gamma\left\|s_{k}\right\|}{\alpha_{k}}+\left(\frac{u\left(\eta_{2}+\eta_{1}\left\|s_{k}\right\|\right)\left\|s_{k}\right\|+2 v \gamma^{2}}{w \eta_{1}\left\|s_{k}\right\|^{2}}\right)^{2} \frac{\left\|s_{k}\right\|^{2}}{\alpha_{k}^{2}} \\
\leq & \eta_{2} D+2\left(\frac{u\left(\eta_{2}+\eta_{1} D\right) D+2 v \gamma^{2}}{w \alpha_{k} \eta_{1} D}\right) \gamma+\left(\frac{u\left(\eta_{2}+\eta_{1} D\right) D+2 v \gamma^{2}}{w \alpha_{k} \eta_{1} D}\right)^{2}=\psi_{2}^{2}
\end{aligned}
$$

Thus $\left\|d_{k+1}\right\|^{2} \leq \psi_{2}^{2}$

$$
\Rightarrow \frac{1}{\left\|d_{k+1}\right\|^{2}} \geq \frac{1}{\psi_{2}^{2}}
$$

Summation this inequality for all $k \geq 1$

$$
\sum_{k \geq 1} \frac{1}{\left\|d_{k+1}\right\|} \geq \sum_{k \geq 1} \frac{1}{\psi_{2}^{2}}=\infty
$$

Using Theorem (3.7.1), hence $\liminf _{k \rightarrow \infty}\left\|g_{k}\right\|=0$ 
But $f$ is uniformly convex (3.37) therefore $\lim _{k \rightarrow \infty} g_{k}=0$.

Case (3) from equation (7) we have also:

$$
\left|\beta_{k+1}^{\text {new3 }}\right| \leq \frac{u\left\|g_{k+1}\right\|^{2}+u\left\|g_{k}\right\|^{2}+2 v\left\|g_{k+1}\right\|\left\|g_{k}\right\|}{\frac{w}{\alpha}\left\|s_{k}\right\|\left\|y_{k}\right\|}
$$

But the gradient $g$ is Lipschitz and $\mathrm{f}$ is uniformly convex then we get

$$
\mu\left\|s_{k}\right\| \leq\left\|y_{k}\right\| \leq L\left\|s_{k}\right\|
$$

Therefore

$$
\begin{aligned}
\left|\beta_{k}^{\text {new3 }}\right| \leq & \frac{u\left\|g_{k+1}\right\|^{2}+u\left\|g_{k}\right\|^{2}+2 v\left\|g_{k+1}\right\|\left\|g_{k}\right\|}{\frac{w}{\alpha} \mu\left\|s_{k}\right\|^{2}} \\
& \leq \frac{u\left(\eta_{2}+\eta_{1}\left\|s_{k}\right\|\right)\left\|s_{k}\right\|+2 v \gamma \gamma}{\frac{w}{\alpha} \mu D^{2}}=\frac{\alpha_{k} u\left(\eta_{2}+\eta_{1} D\right) D+2 v \alpha_{k} \gamma^{2}}{w \mu D^{2}}
\end{aligned}
$$

Then

$$
\left|\beta_{k}^{\text {new } 3}\right| \leq \frac{\alpha_{k} u\left(\eta_{2}+\eta_{1} D\right) D+2 v \alpha_{k} \gamma^{2}}{w \mu D^{2}}
$$

Again we know that

$$
\begin{aligned}
& d_{k+1}=-g_{k+1}+\beta_{k}^{\text {new3 }} d_{k} \\
& \begin{array}{c}
\left\|d_{k+1}\right\|^{2}=\left\|-g_{k+1}+\beta_{k}^{\text {new } 3} d_{k}\right\|^{2} \\
\leq\left\|g_{k+1}\right\|^{2}+2 \mid \beta_{k}^{\text {new } 3}\left\|g_{k+1}\right\| d_{k}\left\|+\left(\beta_{k}^{\text {new } 3}\right)^{2}\right\| d_{k} \|^{2} \\
\leq \eta_{2}\left\|s_{k}\right\|+2\left(\frac{\alpha_{k} u\left(\eta_{2}+\eta_{1} D\right) D+2 v \alpha_{k} \gamma^{2}}{w \mu D^{2}}\right) \frac{\gamma}{\alpha_{k}}\left\|s_{k}\right\|+\left(\frac{\alpha_{k} u\left(\eta_{2}+\eta_{1} D\right) D+2 v \alpha_{k} \gamma^{2}}{w \mu D^{2}}\right)^{2} \frac{1}{\alpha_{k}^{2}}\left\|s_{k}\right\|^{2}
\end{array} \\
& \leq \eta_{2} D+2\left(\frac{u\left(\eta_{2}+\eta_{1} D\right) D+2 v \gamma^{2}}{w \mu D}\right) \gamma+\left(\frac{u\left(\eta_{2}+\eta_{1} D\right) D+2 v \gamma^{2}}{w \mu D}\right)^{2}=\psi_{3}^{2} \\
& \text { Thus } \Rightarrow \frac{1}{\left\|d_{k+1}\right\|^{2} \leq \psi_{3}{ }^{2}} \geq \frac{1}{\psi_{3}{ }^{2}}
\end{aligned}
$$

Summation this inequality for all $\quad k \geq 1$

$$
\sum_{k \geq 1} \frac{1}{\left\|d_{k+1}\right\|^{2}} \geq \sum_{k \geq 1} \frac{1}{\psi_{3}{ }^{2}}=\infty
$$

Using theorem (3.2.1), hence $\liminf _{k \rightarrow \infty}\left\|g_{k}\right\|=0$

But $f$ is uniformly convex (3.37), therefore $\lim _{k \rightarrow \infty} g_{k}=0$

\subsection{Global Convergence Property For General Nonlinear Functions}

For general nonlinear functions, the convergence analysis of our algorithms exploits insights developed by Gilbert and Nocedal [9]; Dai and Liao [8] and Hager and Zhang [10]. The global convergence proof of (New1, New2, New3) CG-algorithms 
is based on the Zoutendijk condition as showed in Lemma (3.5). combined with the analysis showing that the descent property holds and $\left\|d_{k}\right\|$ is bounded. Suppose that the level set $\mathrm{S}$ is bounded and the function $\mathrm{f}$ is bounded from below.

3.7.1 Theorem Let Assumption $(\mathrm{H})$ hold, $d_{k+1}$ is descent direction and $\alpha_{k}$ is obtained by line search satisfies strong wolf line search, $c>0,0<u, v, w \leq 1$ also constants $\gamma>0$ such that $\left\|g_{k}\right\| \leq \gamma$ as in (10). Then the algorithm (2.1) satisfies, either $g_{k}=0$ for some k or(44) s. t. $\liminf _{k \rightarrow \infty}\left\|g_{k}\right\|=0$

Proof We will prove this theorem by using contradiction, then assume that the result is not true, So there exists a constant s. t. $\left\|g_{k}\right\| \geq \xi, \forall k \geq 1$

Case (1) we begin with direction of CG-method contains the parameter $\beta_{k}^{\text {newl }}$

$$
\begin{aligned}
\left\|d_{k+1}\right\| & =\left\|-g_{k+1}+\frac{u\left(\left\|g_{k+1}\right\|^{2}+\left\|g_{k}\right\|^{2}\right)-2 v g_{k+1}{ }^{T} g_{k}}{-w d_{k}{ }^{T} g_{k}} d_{k}\right\| \\
& \leq\left\|g_{k+1}\right\|+\frac{u\left\|g_{k+1}\right\|^{2}+u\left\|g_{k}\right\|^{2}+2 v\left\|g_{k+1}\right\| g_{k} \|}{w\left\|d_{k}\right\|\left\|g_{k}\right\|}\left\|d_{k}\right\| \\
& \leq \gamma+\frac{u \gamma^{2}+u \gamma^{2}+2 v \gamma^{2}}{w \gamma}=\zeta_{1}
\end{aligned}
$$

Thus:

$\left\|d_{k+1}\right\| \leq \zeta_{1}$

Since the level set $\mathrm{S}$ is bounded and the function $f$ is bounded below using \{Lemma (3.3) and Lemma (3.4)\}, we get:

$$
0 \leq \sum_{k \geq 1} \frac{\left(g_{k}{ }^{T} d_{k}\right)^{2}}{\left\|d_{k}\right\|^{2}}<\infty
$$

Combining with sufficient descent condition (15) yields:

$$
\sum_{k \geq 1} \frac{\xi^{4}}{\left\|d_{k}\right\|^{2}} \leq \sum_{k \geq 1} \frac{\left\|g_{k}\right\|^{4}}{\left\|d_{k}\right\|^{2}} \leq \frac{1}{c_{1}} \sum_{k \geq 1} \frac{\left(g_{k}{ }^{T} d_{k}\right)^{2}}{\left\|d_{k}\right\|^{2}}<+\infty
$$

Using the above inequality with (3.46) which contradiction to (40). Hence (41) holds s.t. $\liminf _{k \rightarrow \infty}\left\|g_{k}\right\|=0$

Case (2) with direction of CG-method contains the parameter $\beta_{k}^{\text {new } 2}$

$$
\begin{aligned}
\left\|d_{k+1}\right\| & =\left\|-g_{k+1}+\left(\frac{u\left(\left\|g_{k+1}\right\|^{2}+\left\|g_{k}\right\|^{2}\right)-2 v g_{k+1}{ }^{T} g_{k}}{w\left\|g_{k}\right\|^{2}}\right) \frac{1}{\alpha_{k}} s_{k}\right\| \\
& \leq\left\|g_{k+1}\right\|+\frac{u\left\|g_{k+1}\right\|^{2}}{\alpha_{k} w\left\|g_{k}\right\|^{2}}\left\|s_{k}\right\|+\frac{u\left\|g_{k}\right\|^{2}}{\alpha_{k} w\left\|g_{k}\right\|^{2}}\left\|s_{k}\right\|+\frac{2 v\left\|g_{k+1}\right\| g_{k} \|}{\alpha_{k} w\left\|g_{k}\right\|^{2}}\left\|s_{k}\right\|
\end{aligned}
$$




$$
\begin{aligned}
& \leq \gamma+\frac{u \gamma^{2}}{\alpha_{k} w \gamma^{2}}\left\|s_{k}\right\|+\frac{u}{\alpha_{k} w}\left\|s_{k}\right\|+\frac{2 v \gamma \gamma}{\alpha_{k} w \gamma^{2}}\left\|s_{k}\right\| \\
& =\gamma+\left(\frac{2(u+v)}{\alpha_{k} w}\right)\left\|s_{k}\right\| \\
& \leq \gamma+2 D\left(\frac{u+v}{\alpha_{k} w}\right)=\zeta_{2}
\end{aligned}
$$

Thus:

$\left\|d_{k+1}\right\| \leq \zeta_{2}$

Since the level set $\mathrm{S}$ is bounded and the function $f$ is bounded below using lemmas (3.2) and (3.3), we get:

$$
0 \leq \sum_{k \geq 1} \frac{\left(g_{k}{ }^{T} d_{k}\right)^{2}}{\left\|d_{k}\right\|^{2}}<\infty
$$

Combining with sufficient descent condition (17), s. t.

$$
\begin{gathered}
-d_{k}{ }^{T} g_{k} \geq c_{2}\left\|g_{k}\right\|^{2}>0 \\
\sum_{k \geq 1} \frac{\xi^{4}}{\left\|d_{k}\right\|^{2}} \leq \sum_{k \geq 1} \frac{\left\|g_{k}\right\|^{4}}{\left\|d_{k}\right\|^{2}} \leq \frac{1}{c_{2}} \sum_{k \geq 1} \frac{\left(g_{k}{ }^{T} d_{k}\right)^{2}}{\left\|d_{k}\right\|^{2}}<+\infty
\end{gathered}
$$

Using the above inequality with (47) which contradiction to (40). Hence (3.34) holds s.t. $\liminf _{k \rightarrow \infty}\left\|g_{k}\right\|=0$

Case( 3) with direction of CG-method contains the parameter $\beta_{k}^{\text {new3 }}$

$$
\begin{aligned}
\left\|d_{k+1}\right\| & =\left\|-g_{k+1}+\left(\frac{u\left(\left\|g_{k+1}\right\|^{2}+\left\|g_{k}\right\|^{2}\right)-2 v g_{k+1}{ }^{T} g_{k}}{\frac{w}{\alpha_{k}} s_{k}{ }^{T} y_{k}}\right) \frac{1}{\alpha_{k}} s_{k}\right\| \\
& \leq\left\|g_{k+1}\right\|+\frac{u\left\|g_{k+1}\right\|^{2}}{w\left\|s_{k}\right\|\left\|y_{k}\right\|}\left\|s_{k}\right\|+\frac{u\left\|g_{k}\right\|^{2}}{w\left\|s_{k}\right\|\left\|y_{k}\right\|}\left\|s_{k}\right\|+\frac{2 v\left\|g_{k+1}\right\|\left\|g_{k}\right\|}{w\left\|s_{k}\right\|\left\|y_{k}\right\|}\left\|s_{k}\right\|
\end{aligned}
$$

But: $\left\|y_{k}\right\|=\left\|g_{k+1}-g_{k}\right\| \leq\left\|g_{k+1}\right\|+\left\|g_{k}\right\| \leq 2 \gamma$ and from equation (45), we obtain

$$
\begin{aligned}
& \Rightarrow 2 \xi \leq\left\|y_{k}\right\| \leq 2 \gamma \\
& \leq\left\|g_{k+1}\right\|+\frac{u\left\|g_{k+1}\right\|^{2}}{2 w \xi\left\|s_{k}\right\|}\left\|s_{k}\right\|+\frac{u\left\|g_{k}\right\|^{2}}{2 w \xi\left\|s_{k}\right\|}\left\|s_{k}\right\|+\frac{2 v\left\|g_{k+1}\right\|\left\|g_{k}\right\|}{2 w \xi\left\|s_{k}\right\|}\left\|s_{k}\right\| \\
& \quad \leq\left\|g_{k+1}\right\|+\frac{u\left\|g_{k+1}\right\|^{2}}{2 w \xi}+\frac{u\left\|g_{k}\right\|^{2}}{2 w \xi}+\frac{2 v\left\|g_{k+1}\right\|\left\|g_{k}\right\|}{2 w \xi}
\end{aligned}
$$

Therefore

$\left\|d_{k+1}\right\| \leq \gamma+\frac{u \gamma^{2}+u+2 v \gamma \gamma}{2 w \xi}=\zeta_{3}$

Thus

$\left\|d_{k+1}\right\| \leq \zeta_{3}$ 
Since the level set $\mathrm{S}$ is bounded and the function $f$ is bounded below using lemmas (3.2) and (3.3), we get:

$$
0 \leq \sum_{k \geq 1} \frac{\left(g_{k}{ }^{T} d_{k}\right)^{2}}{\left\|d_{k}\right\|^{2}}<\infty
$$

Combining with sufficient descent condition (21), s. t.

$$
\sum_{k \geq 1} \frac{\xi^{4}}{\left\|d_{k}\right\|^{2}} \leq \sum_{k \geq 1} \frac{\left\|g_{k}\right\|^{4}}{\left\|d_{k}\right\|^{2}} \leq \frac{1}{c_{3}} \sum_{k \geq 1} \frac{\left(g_{k}{ }^{T} d_{k}\right)^{2}}{\left\|d_{k}\right\|^{2}}<+\infty
$$

Using the above inequality with (48) which contradiction to (40). Hence (41) holds s.t. $\liminf _{k \rightarrow \infty}\left\|g_{k}\right\|=0$.

\section{Numerical Results}

The main work of this section is to report the performance of the new methods on a set of test problems. the codes were written in Fortran and in double precision arithmetic. All the tests were performed on a PC. Our experiments were performed on a set of 35-nonlinear unconstrained problems that have second derivatives available. These test problems are contributed in CUTE [7] and their details are given in the Appendix. for each test function we have considered 10 numerical experiments with number of variable $n=100,200, \ldots \ldots, 1000$. In order to assess the reliability of our new proposed methods, we have tested them against FR and PR classical CG-methods using the same test problems. All these methods terminate when the following stopping criterion is met.

$\left\|g_{k+1}\right\| \leq 1 \times 10^{-5}$

We also force these routines stopped if the iterations exceed 1000 or the number of function evaluations reach 2000 without achieving the minimum. We use $\delta=10^{-4}, \sigma=0.1$ in the Wolfe line search routine. Tables (4.1); (4.2) and (4.3) compare some numerical result for (New1, New2 and New3) CG-methods against FR and PR CG-methods respectively, these tables indicate for (n) as a dimension of the problem;(NOI) number of iterations; (NOFG) number of function and gradient evaluation;(Time) the total time required to complete the evaluation process for each test problem. In Tables $(4.4,4.5,4.6)$ we have compared the percentage performance of the new and FR\&PR methods taking over all the tools as $100 \%$. In order to summarize our numerical results, we have concerned only on the total of different dimensions $n=$ $100,200, \ldots \ldots, 10000$, for all tools used in these comparisons.

Table 4.1 Comparison between new1 and classical FR and PR CG-methods for the total of $\mathrm{n}$ different dimensions $\mathrm{n}=100,200, \ldots \ldots, 1000$ for each test problems with parameter $\left(\mathrm{u}=0.3, \mathrm{v}=0.3, \mathrm{w}=0.8 ; \varepsilon=1 * 10^{-5}\right)$.

Table 4.2 Comparison between new2 and classical FR and PR CG-methods for the total of $n$ different dimensions $n=100,200, \ldots \ldots, 1000$ for each test problems with parameter $\left(\mathrm{u}=0.3, \mathrm{v}=0.7, \mathrm{w}=0.8 ; \varepsilon=1 * 10^{-5}\right)$.

Table 4.3 Comparison between new3 and classical FR and PR CG-methods for the total of $n$ different dimensions $n=100,200, \ldots \ldots, 1000$ for each test problems with parameter $\left(\mathrm{u}=0.4, \mathrm{v}=0.4, \mathrm{w}=0.1 ; \varepsilon=1 * 10^{-5}\right)$. 
Table (4.1) Comparison between New1, FR and PR CG-methods for the total of $n$ different dimensions $n=100,200, \ldots, \ldots 1000$, for each test problem $\left(u=0.4, v=0.6, w=0.8, \varepsilon=1 * 10^{-5}\right)$.

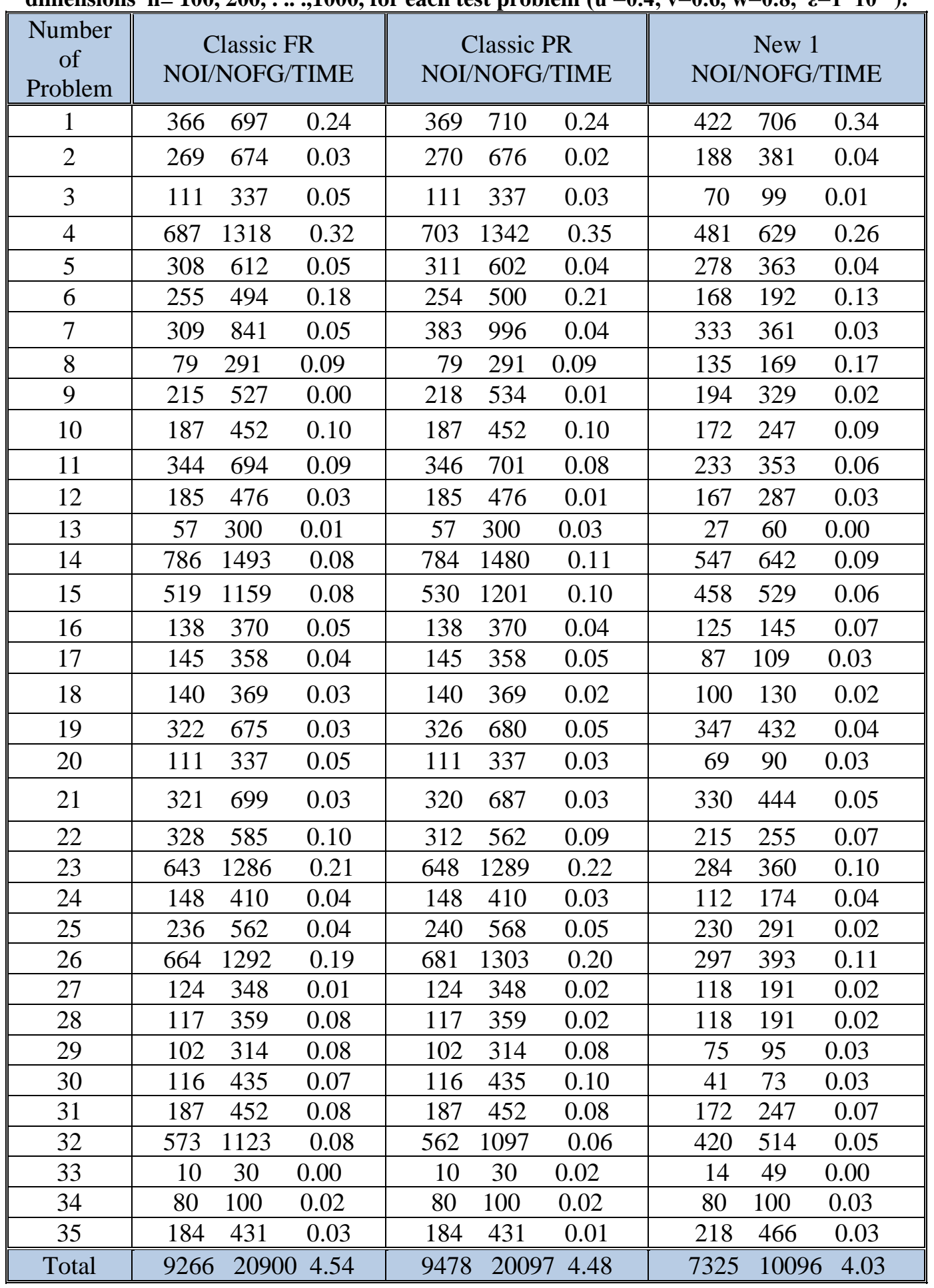


Table (4.2) Comparison between New2, FR and PR CG-methods for the total of $\mathbf{n}$ different dimensions $n=100,200, \ldots \ldots, 1000$, for each test problem $\left(u=0.3, v=0.4, w=0.5, \varepsilon=1 * 10^{-5}\right)$.

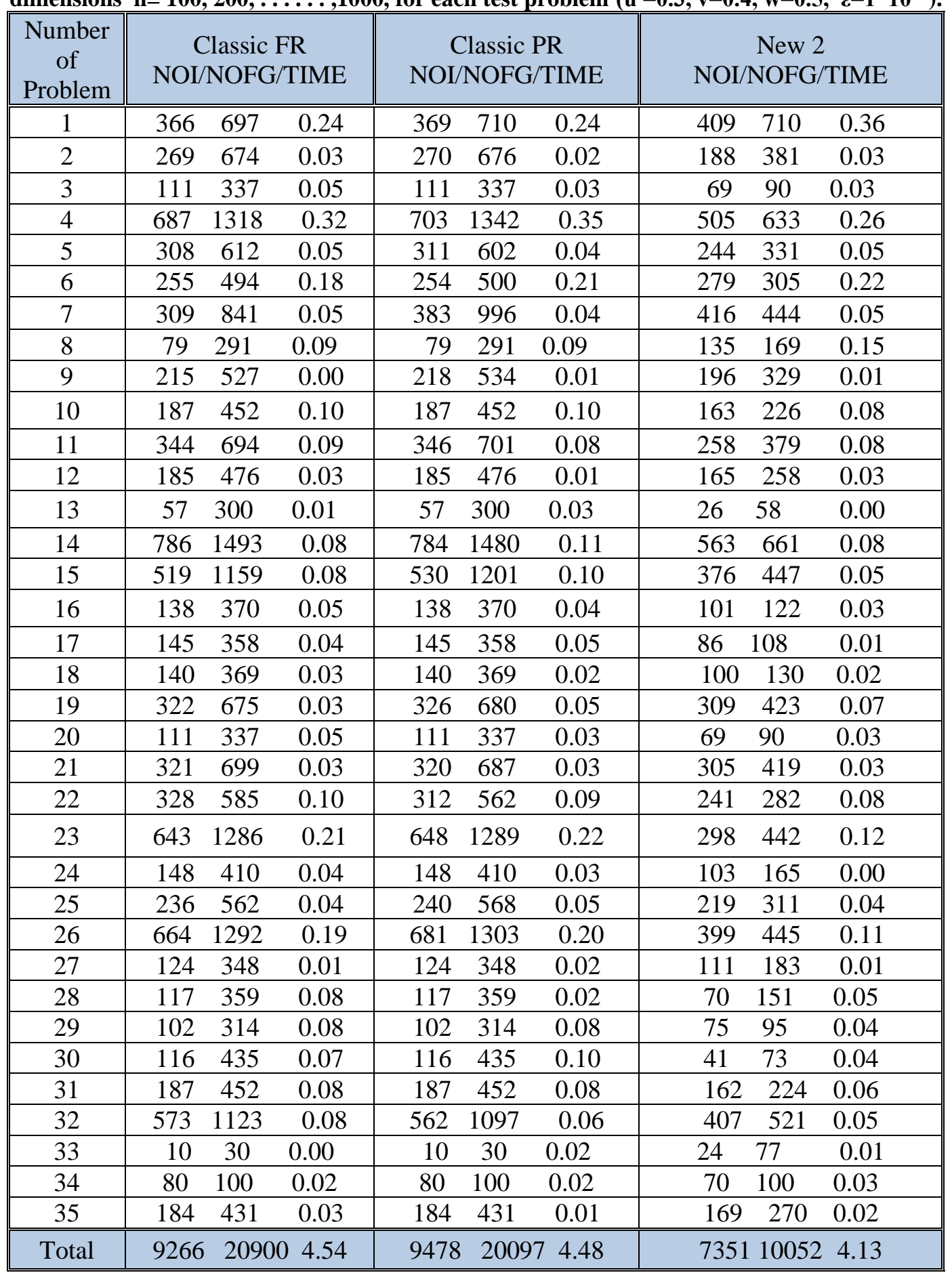


Table (4.3) Comparison between New3, FR and PR CG-methods for the total of $n$ different dimensions $n=100,200, \ldots \ldots, 1000$, for each test problem $\left(u=0.4, v=0.6, w=0.8, \varepsilon=1 * 10^{-5}\right)$.

\begin{tabular}{|c|c|c|c|c|c|c|c|}
\hline \multirow{2}{*}{$\begin{array}{c}\begin{array}{c}\text { Number } \\
\text { of } \\
\text { Problem }\end{array} \\
1\end{array}$} & \multicolumn{2}{|c|}{$\begin{array}{c}\text { Classic FR } \\
\text { NOI/NOFG/TIME }\end{array}$} & \multicolumn{2}{|c|}{$\begin{array}{c}\text { Classic PR } \\
\text { NOI/NOFG/TIME }\end{array}$} & \multicolumn{3}{|c|}{$\begin{array}{c}\text { New } 3 \\
\text { NOI/NOFG/TIME }\end{array}$} \\
\hline & $\begin{array}{ll}366 & 697\end{array}$ & 0.24 & 369710 & 0.24 & 417 & 690 & 0.38 \\
\hline 2 & 674 & 0.03 & $270 \quad 676$ & 0.02 & 191 & 384 & 0.01 \\
\hline 3 & 111337 & 0.05 & $111 \quad 337$ & 0.03 & 69 & 90 & 0.03 \\
\hline 4 & $\begin{array}{ll}687 & 1318\end{array}$ & 0.32 & $\begin{array}{ll}703 & 1342\end{array}$ & 0.35 & 461 & 613 & 0.26 \\
\hline 5 & $308 \quad 612$ & 0.05 & 311602 & 0.04 & 284 & 374 & 0.05 \\
\hline 6 & $255 \quad 494$ & 0.18 & 254500 & 0.21 & 141 & 164 & 0.09 \\
\hline 7 & 841 & 0.05 & $383 \quad 996$ & 0.04 & 382 & 411 & 0.05 \\
\hline 8 & $\begin{array}{ll}79 & 291\end{array}$ & 0.09 & $\begin{array}{ll}79 & 291\end{array}$ & 0.09 & 128 & 162 & 0.14 \\
\hline 9 & $\begin{array}{ll}215 & 527 \\
\end{array}$ & 0.00 & $218 \quad 534$ & 0.01 & 190 & 309 & 0.04 \\
\hline 10 & 452 & 0.10 & $187 \quad 452$ & 0.10 & 174 & 246 & 0.08 \\
\hline 11 & 694 & 0.09 & $346 \quad 701$ & 0.08 & 217 & 329 & 0.06 \\
\hline 12 & 476 & 0.03 & $185 \quad 476$ & 0.01 & 158 & 292 & 0.00 \\
\hline 13 & $\begin{array}{ll}57 & 300\end{array}$ & 0.01 & $\begin{array}{ll}57 & 300\end{array}$ & 0.03 & 26 & 58 & 0.00 \\
\hline 14 & $\begin{array}{ll}786 & 1493 \\
\end{array}$ & 0.08 & $\begin{array}{ll}784 & 1480 \\
\end{array}$ & 0.11 & 554 & 644 & 0.08 \\
\hline 15 & $519 \quad 1159$ & 0.08 & $\begin{array}{ll}530 & 1201 \\
\end{array}$ & 0.10 & 465 & 536 & 0.08 \\
\hline 16 & $138 \quad 370$ & 0.05 & $\begin{array}{ll}38 & 370\end{array}$ & 0.04 & 125 & 145 & 0.03 \\
\hline 17 & 358 & 0.04 & $145 \quad 358$ & 0.05 & 87 & 109 & 0.01 \\
\hline 18 & 369 & 0.03 & $140 \quad 369$ & 0.02 & 100 & 130 & 0.03 \\
\hline 19 & 675 & 0.03 & $326 \quad 680$ & 0.05 & 331 & 435 & 0.06 \\
\hline 20 & 337 & 0.05 & $111 \quad 337$ & 0.03 & 69 & 90 & 0.03 \\
\hline 21 & 699 & 0.03 & $320 \quad 687$ & 0.03 & 315 & 395 & 0.05 \\
\hline 22 & 585 & 0.10 & $312 \quad 562$ & 0.09 & 219 & 246 & 0.08 \\
\hline 23 & $\begin{array}{ll}643 \quad 1286\end{array}$ & 0.21 & $\begin{array}{ll}648 & 1289\end{array}$ & 0.22 & 277 & 356 & 0.12 \\
\hline 24 & $148 \quad 410$ & 0.04 & $148 \quad 410$ & 0.03 & 112 & 174 & 0.00 \\
\hline 25 & $236 \quad 562$ & 0.04 & $240 \quad 568$ & 0.05 & 254 & 372 & 0.04 \\
\hline 26 & $\begin{array}{ll}664 & 1292\end{array}$ & 0.19 & $\begin{array}{ll}681 & 1303\end{array}$ & 0.20 & 303 & 365 & 0.11 \\
\hline 27 & $124 \quad 348$ & 0.01 & $124 \quad 348$ & 0.02 & 130 & 189 & 0.01 \\
\hline 28 & 359 & 0.08 & $117 \quad 359$ & 0.02 & 70 & 151 & 0.03 \\
\hline 29 & 314 & 0.08 & $102 \quad 314$ & 0.08 & 75 & 95 & 0.05 \\
\hline 30 & 435 & 0.07 & $116 \quad 435$ & 0.10 & 41 & 73 & 0.05 \\
\hline 31 & $\begin{array}{ll}187 & 452\end{array}$ & 0.08 & $\begin{array}{ll}187 \quad 452 \\
\end{array}$ & 0.08 & 174 & 246 & 0.08 \\
\hline 32 & $\begin{array}{ll}573 & 1123 \\
\end{array}$ & 0.08 & $\begin{array}{ll}562 & 1097\end{array}$ & 0.06 & 423 & 521 & 0.06 \\
\hline 33 & $10 \quad 30$ & 0.00 & $10 \quad 30$ & 0.02 & 22 & 70 & 0.00 \\
\hline 34 & $\begin{array}{ll}80 & 100\end{array}$ & 0.02 & 100 & 0.02 & 70 & 100 & 0.03 \\
\hline 35 & $184 \quad 431$ & 0.03 & $\begin{array}{ll}184 & 431\end{array}$ & 0.01 & 180 & 270 & 0.02 \\
\hline Total & 926620900 & 4.54 & $\begin{array}{ll}9478 & 20097\end{array}$ & 74.48 & 7234 & 98 & 4.02 \\
\hline
\end{tabular}

Percentage Performance of each New algorithm against $100 \%$ of Fletcher-Reeves (FR), Polak- Ribiere (PR), algorithms respectively, as follows in Tables (4.4), (4.5), (4.6). 
Table (4.4) Performance of the New1 algorithm against $100 \%$ of Fletcher-Reeves (FR) and Polak- Ribiere (PR) algorithm, as followed in Table (4.1).

\begin{tabular}{|c||c|c||c|c|c||}
\hline Tools & FR & New1 & & PR & New1 \\
\hline \hline NOI & $100 \%$ & $79.052 \%$ & & $100 \%$ & $77.284 \%$ \\
\hline \hline NOFG & $100 \%$ & $53.549 \%$ & & $100 \%$ & $50.236 \%$ \\
\hline Time & $100 \%$ & $57.202 \%$ & & $100 \%$ & $89.955 \%$ \\
\hline
\end{tabular}

Table (4.5) Performance of the New2 algorithm against 100\% of Fletcher-Reeves (FR) and Polak- Ribiere (PR) algorithm, as followed in Table (4.2).

\begin{tabular}{|c|c||c||c|c|c||}
\hline Tools & FR & New2 & & PR & New2 \\
\hline \hline NOI & $100 \%$ & $79.333 \%$ & & $100 \%$ & $77.558 \%$ \\
\hline \hline NOFG & $100 \%$ & $48.059 \%$ & \\
\hline \hline Time & $100 \%$ & $90.969 \%$ & & $100 \%$ & $50.017 \%$ \\
\cline { 1 - 1 } \cline { 5 - 6 } & $100 \%$ & $92.187 \%$ \\
\hline
\end{tabular}

Table (4.6) Performance of the New3 algorithm against $100 \%$ of Fletcher-Reeves (FR) and Polak-Ribiere (PR) algorithm, as followed in Table (4.3).

\begin{tabular}{|c|c|c|c|c|}
\hline Tools & FR & New3 & PR & New3 \\
\hline NOI & $100 \%$ & $78.070 \%$ & $100 \%$ & $76.324 \%$ \\
\hline NOFG & $100 \%$ & $47.052 \%$ & $100 \%$ & $48.632 \%$ \\
\hline Time & $100 \%$ & $88.546 \%$ & $100 \%$ & $89.732 \%$ \\
\hline
\end{tabular}

From the above tables we have concluded that the first new algorithm beats FR and PR CG-algorithms in all NOI; NOFG and Time in about (10-50)\% percentages. However, the second new algorithm is also beats FR and PR CG-algorithms in all NOI; NOFG and Time in about (8-52)\% percentages. Also the third new algorithm is also beats FR and PR CGalgorithms in all NOI; NOFG and Time in about (11-53)\% percentages.

\section{Conclusions}

In this paper, by using scaling parameter idea, we have proposed three new scaled CG-methods with (2.1), (2.2) and (2.3) for $\beta_{k}$, under some assumptions. Our CGmethods have been shown to be globally convergent for uniformly convex and general functions respectively. Some numerical results have been reported against BA1; BA2; BA3; FR and PRCG-algorithms which showed the effectiveness of our new proposed CG-algorithms with the scalars $\mathrm{u}, \mathrm{v}$ and $\mathrm{w}$.

\section{Appendix}

The details of the 35-test functions used are: 1-Extended Trigonometric Function. 2-Extended Penalty Function. 3-Raydan2 Function. 4-Hager Function. 5-Generalized Tridiagonal-1 Function. 6-Extended Three Exponential Function. 7-Diagonal 4 Function. 8-Diagonal5 Function. 9-Extended Himmelblau Function. 10-Generalized PSC1 Function. 11- Extended Block Diagonal BD1 Function. 12-Extended Quadratic Penalty QP1 Function. 13-Extended Quadratic QF2 Function. 14- Extended EP1 Function.15-Extended Tri-diagonal 2 Function. 16- DIXMAANA Function. 17DIXMAANB Function. 18- DIXMAANC Function. 19-EDENSCH Function. 20DIAGONAL 6 Function. 21-ENGVALI Function. 22-DENSCHNA Function. 23DENSCHNC Function. 24-DENSCHNB Function. 25-DENSCHNF Function. 26Extended Block-Diagonal BD2 Function. 27-Generalized quadratic GQ1 Function. 28DIAGONAL 7 Function. 29- DIAGONAL 8 Function. 30- Full Hessian Function. 31-SINCOS Function. 32- Generalized quadratic GQ2 Function. 33-ARGLINB Function. 34-HIMMELBG Function. 35-HIMMELBH Function. 


\section{$\underline{\text { REFERENCES }}$}

[1] Al-Bayati Y., Jassim A. and Abbo K., (2010)," New scaled CG methods based on Newton direction for solving unconstrained optimization problems ". Australian J. of Basic and Applied Sciences, 1190-1202.

[2] Al-Bayati A. Y. and Ban A. Matras, (2008), "A New Five Parameter Family for CG-Methods ", Allepo Conf. 10 (1).

[3] Al-Bayati A.Y. and Al-Assady N. H., (1986)," Conjugate Gradient Algorithms for Unconstrained Optimization ", Technical Report, No. (1/86), School of Computer Studies, Leeds University, U.K.

[4] Al-Baali M., (1985), "Descent Property and Global Convergence of the FletcherReeves Method with Inexact Line Search ", IMA Journal of Numerical Analysis, 5, pp.121-124..

[5] Andrei N. (2009), "Accelerated of conjugate gradient algorithm for unconstrained optimization", Applied Mathematical Computational, 213, pp.361-369.

[6] Andrei N., (2009)," Hybrid Conjugate Gradient Algorithm for Unconstrained Optimization", J. Optim. Theory Appl. 141, pp. 249-264.

[7] Bongartz K. E., Conn A.R., Gould N.I.M. and Toint P.L., CUTE, (1995), "constrained and unconstrained testing environments". ACM Trans. Math. Software, 21, pp. 123-160.

[8] Dai Y.H. and Liao L.Z., (2001), "New conjugacy conditions and related nonlinear conjugate gradient methods", Applied. Mathematic. Optimization. 43, 87-101.

[9] Dai Y. H. and Yuan Y., (1998), "Nonlinear Conjugate Gradient Methods", Shanghai Scientific and Technical Publishers, Shanghai.

[10] Gilbert J.C. and Nocedal J. ,(1992), "Global convergence properties of conjugate gradient methods for optimization". SIAM J. Optimization. 2, pp. 21-42.

[11] Hager W.W. and Zhang H., (2005), "A new conjugate gradient method with guaranteed descent and an efficient line search", SIAM J. Optim. 16, pp.170-192.

[12] Hager W.W. and Zhang H., (2005), "A survey of nonlinear conjugate gradient methods", Department of Mathematics, University of Florida, Gainesville, FL, pp. 32611- 8105 .

[13] Powell M. J. D., (1984), "Non-convex Minimization Calculations and the Conjugate Gradient Method", Lecture Notes in Mathematics, 1066, pp.121-141. 
[14] Powell M.J.D., (1977), "Restart procedures for the conjugate gradient method", Mathematical Programming, 12, pp. 241-254.

[15] Sun W. and Yuan Y., (1999), "Theory and Methods of Optimization", Science Press of

China, Beijing.

[16] Yabe H. and Takano M., (2004), "Global Convergence Properties of Nonlinear Conjugate Gradient Methods with Modified Secant Condition", Computational Optimization and Applications, 28, 203-225.

[17] Zhang L. and Zhou W. J., (2008), "Two Descent Hybrid Conjugate Gradient Methods for Optimization", Journal of Computational and Applied Mathematics, 216, pp. 251-264.

[18] Zoutendijk G., (1970), "Nonlinear programming, computational methods in integer and nonlinear programming", North Holland Amsterdam, pp. 37-86. 